\title{
EDITORIAL
}

\section{Investigación en salud pública: compromiso de todos}

Parece promisoria la evolución reciente de la comunicación de investigaciones en salud pública que se realiza en el país. Se amplía la convocatoria de las Jornadas Chilenas de Salud Pública, inscribiéndose una mayor cantidad de presentaciones y advirtiéndose una mejoría de su calidad; reaparecen los Cuadernos Médico Sociales del Colegio Médico y se aprecia un aumento del interés en publicar resultados de investigaciones sobre una variada gama de temas en esta Revista. ¿Es esto suficiente para estar optimistas y sólo debemos limitarnos a esperar que esta tendencia se consolide?

Para responder a esta interrogante, es pertinente examinar la situación por la que atraviesa la investigación en salud pública en el país. En primer lugar, debemos reconocer la inmensa brecha que existe con los paises desarrollados -e incluso con México y Brasil-, la cual supera ampliamente a la observada en otros campos del conocimiento. Esto se objetiva fácilmente con indicadores tales como el reducido número de investigadores formados en salud pública en el país, y la escasa cantidad de publicaciones científicas, incluso si se considera a las revistas no indexadas. Así, el bien ganado prestigio de la salud pública chilena, que se cimienta, entre otros, en el desarrollo de un pensamiento innovador y experiencias exitosas, es insuficientemente conocido y valorado a escala internacional, en gran medida porque muchas experiencias no se han sistematizado, presentado en los foros correspondientes y mucho menos publicado.

Como consecuencia, la competitividad de la salud pública por recursos concursables para investigación disponibles en el ámbito nacional es baja y peor aún, desde hace una década presenta una tendencia claramente decreciente. En los últimos años, se ha intentado romper ese círculo vicioso con un conjunto de iniciativas entre las que destacan la creación de un programa de Doctorado en Salud Pública en el país actualmente en su tercer año de ejecución- y el establecimiento de un fondo específico, el Fondo Nacional de Investigación en Salud, Fonis. En ambas iniciativas es alentador constatar la alta convocatoria que, aún en tiempos de economía de mercado, conserva la salud pública; lo que se refleja en un número creciente de buenos candidatos al doctorado y la presentación de alrededor de 700 proyectos al citado Fondo.

En este contexto, es prioritario impulsar la creación de nuevas instancias de presentación y debate de los variados estudios que se realizan en el país. Las Jornadas Chilenas de Salud Pública y los Congresos de Epidemiología y Administración en Salud han cumplido un papel relevante en tal sentido; sin embargo, creemos que ha llegado el momento de aglutinar fuerzas e impulsar la realización periódica de un evento científico de envergadura -un Congreso Chileno de Salud Pública- que, junto con convocar a una amplia gama de investigadores nacionales, permita un fructifero intercambio de experiencias con figuras internacionales. Una iniciativa de esta envergadura requerirá, sin lugar a dudas, del esfuerzo mancomunado de la comunidad de salud pública, más allá del ámbito académico.

Por otra parte, es responsabilidad de nuestra comunidad, ejercer atenta vigilancia e instar a las autoridades a seguir avanzando en su loable propósito de crear el Fonis como un Fondo para promover la investigación en temas relevantes para la salud en el 
país. Lo deseable es que la asignación de recursos para este Fondo sea progresivamente mayor, de forma que pueda responder más adecuadamente a las expectativas creadas por su gran capacidad de convocatoria.

Sin embargo, no sin cierta preocupación observamos la demora del llamado a un segundo concurso, con relación a los plazos establecidos en el anterior. Esperamos que sea sólo un atraso por razones justificadas. Iniciativas como ésta no pueden surgir para luego descontinuarse, particularmente si han generado una respuesta tal, que supera incluso sus expectativas. 